\title{
A Case of Retroperitoneal Ganglioneuroma Accidentally Discovered by Laparoscopic Right Adrenalectomy
}

\author{
Shunsuke Nomura ${ }^{a}$ Takashi Kawahara ${ }^{a}$ Tappei Takeshima ${ }^{a}$ \\ Takahiro Mitomi $^{a}$ Daiji Takamoto ${ }^{a}$ Tomoe Sawazumi ${ }^{b}$ \\ Yoshiaki Inayamab Hiroji Uemura ${ }^{a}$ \\ aDepartments of Urology and Renal Transplantation, Yokohama City University Medical \\ Center, Yokohama, Japan; ${ }^{b}$ Department of Diagnostic Pathology, Yokohama City University \\ Medical Center, Yokohama, Japan
}

\author{
Keywords \\ Ganglioneuroma $\cdot$ Retroperitoneal tumor · Adenoma
}

\begin{abstract}
A 65-year-old man was referred to our hospital for his right adrenal adenoma. Adrenal vein blood sampling revealed primary hyperaldosteronism, and he was referred to our department for surgical resection of his right adenoma. During the operation, a small nodule was discovered in addition to the adrenal tumor. The pathological diagnosis of this nodule was ganglioneuroma. We herein report a rare case of ganglioneuroma incidentally discovered by laparoscopic right adrenalectomy.

(c) 2020 The Author(s).

Published by S. Karger AG, Basel
\end{abstract}

\section{Introduction}

Glioneuroma is a sympathetic nerve system tumor that develops from the neural crest in the fetal period. Ganglioneuroma in the retroperitoneal cavity is a rare entity. We herein report a case of ganglioneuroma incidentally discovered by laparoscopic right adrenalectomy.

\section{Case Presentation}

A 65-year-old man was referred to our hospital for a right adrenal tumor detected by routine follow-up of diabetes mellitus. He had a history of hypertension and underwent endoscopic tumorectomy for colorectal cancer. Ha had no particular family history. Adrenal vein 


\section{Case Reports in Oncology}

\begin{tabular}{l|l}
\hline Case Rep Oncol 2020;13:764-767 \\
\hline DOI: 10.1159/000507280 & $\begin{array}{l}\text { @ 2020 The Author(s). Published by S. Karger AG, Basel } \\
\text { www.karger.com/cro }\end{array}$ \\
\hline
\end{tabular}

Nomura et al.: A Case of Retroperitoneal Ganglioneuroma Abstract

Fig. 1. Contrast CT findings.
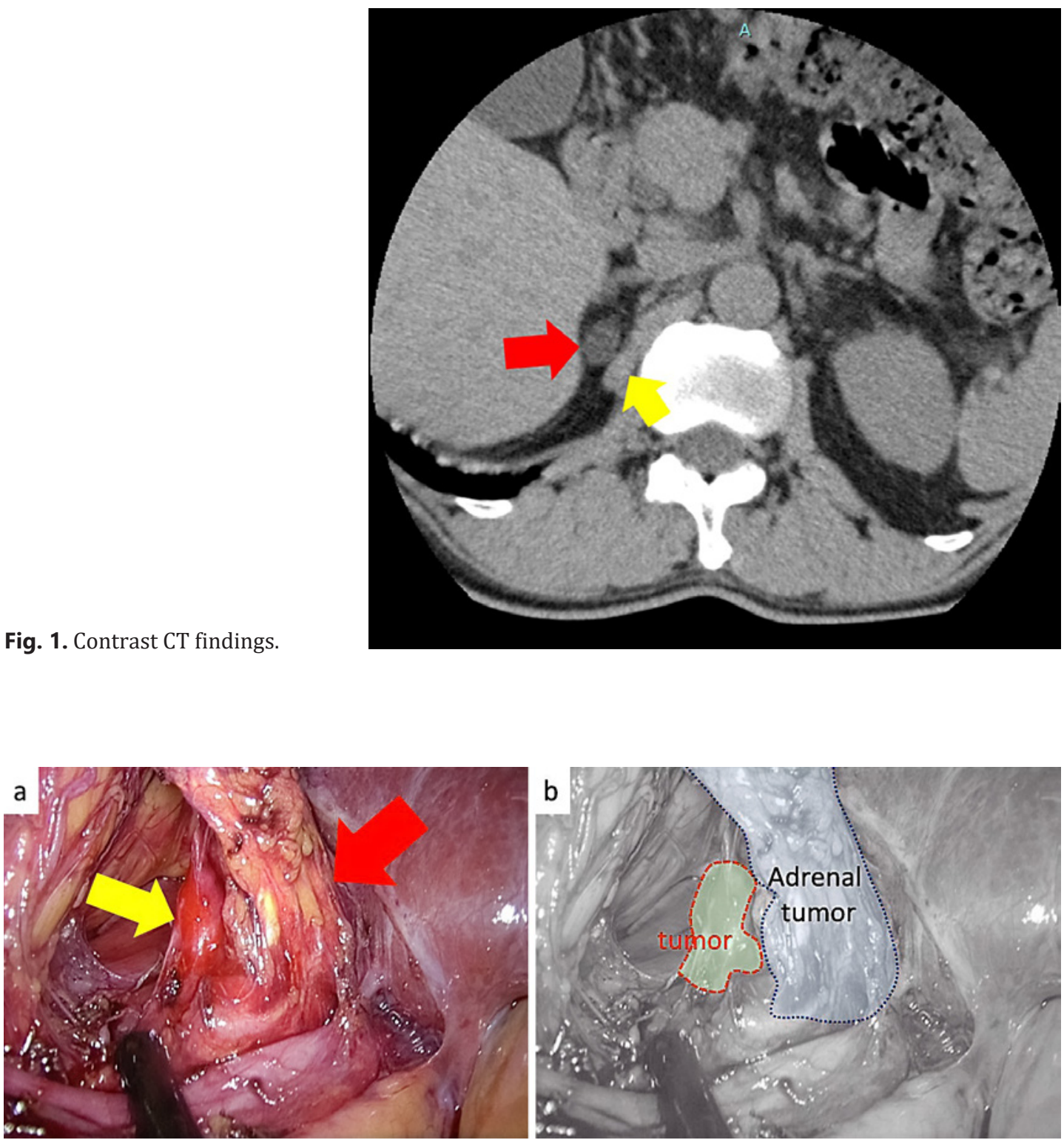

Fig. 2. Intraoperative images. a Yellow arrow: adrenal tumor, red arrow: ganglioneuroma. b Schematic depiction of the intraoperative images.

blood sampling revealed primary hyperaldosteronism, so he was referred to our department for surgical reduction of his adrenal tumor.

Laboratory data showed almost normal findings except for a slightly decreased renin activity $(<0.1 \mathrm{ng} / \mathrm{mL} / \mathrm{h})$. Computed tomography (CT) showed a right adrenal tumor with a 15-mm maximum diameter and lower contrast; the lesion was $5 \mathrm{~mm}$ larger than it had been 2 years ago (Fig. 1). Laparoscopic adenectomy was performed, and during surgery, a small nodule was detected in addition to the adrenal tumor (Fig. 2). We removed this tumor after removing the adrenal tumor. The pathological diagnosis revealed the adrenal tumor to be a cortical adenoma. The nodule was $25 \times 15 \times 8 \mathrm{~mm}$ and white in color. It showed a mixture of ganglion cells and spindle-shaped cells with no immature cells detected. An immunohistochemical analysis revealed positive findings for chromogranin A, S-100, and neuron-specific 

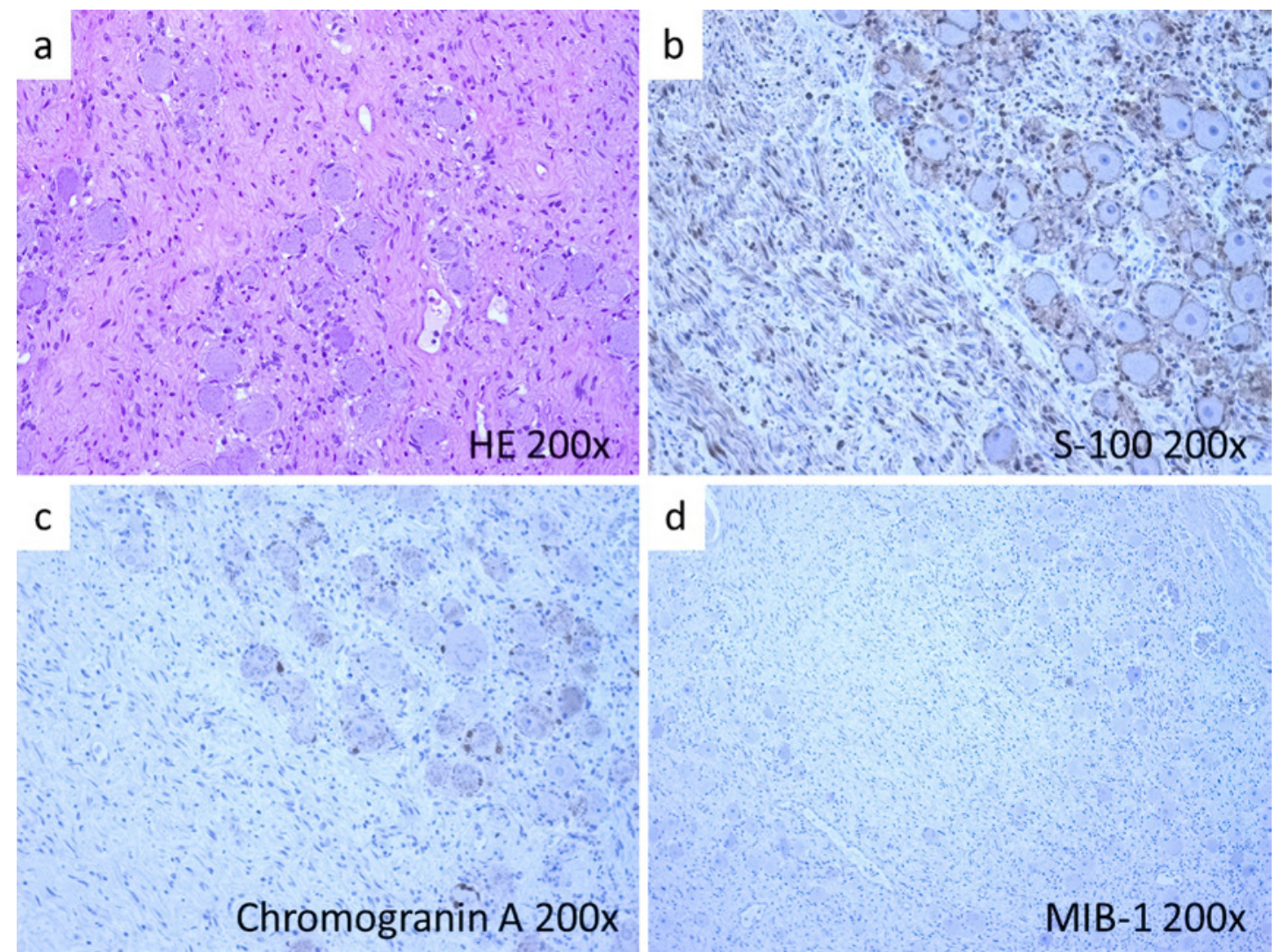

Fig. 3. Pathological findings.

enolase (NSE) in the ganglion cells; the spindle-shaped cells were positive for S-100 and NSE and negative for chromogranin A (Fig. 3).

Based on these findings, ganglioneuroma was diagnosed. He was free from recurrence 3 months postoperatively.

\section{Discussion}

Glioneuroma is a sympathetic nerve system tumor that develops from the neural crest in the fetal period. Sympathetic nerve system tumors are classified as follows: (1) neuroblastoma, (2) ganglioneuroblastoma, and (3) ganglioneuroma [1, 2]. Ganglioneuroma is thought to be a well-differentiated benign tumor. Glioneuroma is a rare tumor accounting for $0.7-1.8 \%$ of all retroperitoneal tumors [3].

Some previous reports have suggested that size follow-up might be an option in tumors of less than $6 \mathrm{~cm}$ without endocrine activity $[4,5]$. However, given that a preoperative diagnosis is difficult for poorly differentiated, transitional-type, or mixed-type tumors, some reports recommend surgical resection $[4,5]$.

This is the first report of a retroperitoneal tumor detected along with a primary hyperaldosteronism adrenal tumor. Due to the low incidence of such tumors and the fact that preoperative CT did not detect it, a preoperative diagnosis was difficult. Fujioka et al. [6] reported 20 cases of ganglioneuroma that were detected at 2 or more sites simultaneously. While glio- 
neuromas are benign, glioneuroma in the present case was found in both the adrenal medulla and lymph node. Despite the low risk of malignant differentiation, follow-up using CT or MRI should be performed.

We encountered a rare case of retroperitoneal ganglioneuroma.

\section{Statement of Ethics}

Written informed consent to participate and for publication was obtained from the patient for ethics approval. A copy of the written consent form is available for review from the Editor-in-Chief of this journal.

\section{Conflict of Interest Statement}

We declare no conflicts of interest.

\section{Funding Sources}

There were no funding sources.

\section{Author Contribution}

S.N. and T.K. drafted the manuscript. T.T., D.T., T.M., T.S., and H.U. performed the experiment.

\section{Availability of Data and Material}

Due to ethical restrictions, the raw data underlying this paper are available upon request to the corresponding author.

\section{References}

1 Scanlan DB. Primary retroperitoneal tumors. J Urol. 1959;81(6):740-5.

2 Hamilton JP, Koop CE. Ganglioneuromas in children. Surg Gynecol Obstet. 1965;121(4):803-12.

3 Enzinger FM, Weiss SW. Soft tissue tumors. 2nd ed. St. Louis: CV Mosby; 1988. pp. 816-35.

4 Bove KE, McAdams AJ. Composite ganglioneuroblastoma. An assessment of the significance of histological maturation in neuroblastoma diagnosed beyond infancy. Arch Pathol Lab Med. 1981;105(6):325-30.

5 Chandrasoma P, Shibata D, Radin R, Brown LP, Koss M. Malignant peripheral nerve sheath tumor arising in an adrenal ganglioneuroma in an adult male homosexual. Cancer. 1986;57(10):2022-5.

6 Fujioka K, Sanuki E, Tanaka Y. A case of retroperitoneal ganglio-neuroma with lymph node metastasis. Jpn J Med Imaging. 1997;16:117-27. 